\title{
Neuronal Activity and Brain-Derived Neurotrophic Factor Regulate the Density of Inhibitory Synapses in Organotypic Slice Cultures of Postnatal Hippocampus
}

\author{
Serge Marty, Rosine Wehrlé, and Constantino Sotelo \\ Institut National de la Santé et de la Recherche Médicale U106, Hôpital de la Salpêtrière, Pavillon de l'Enfance et de \\ l'Adolescence, 75651 Paris cedex 13, France
}

\begin{abstract}
Hippocampal interneurons inhibit pyramidal neurons through the release of the neurotransmitter GABA. Given the importance of this inhibition for the proper functioning of the hippocampus, the development of inhibitory synapses must be tightly regulated. In this study, the possibility that neuronal activity and neurotrophins regulate the density of GABAergic inhibitory synapses was investigated in organotypic slice cultures taken from postnatal day 7 rats.

In hippocampal slices cultured for $13 \mathrm{~d}$ in the presence of the $\mathrm{GABA}_{\mathrm{A}}$ receptor antagonist bicuculline, the density of glutamic acid decarboxylase (GAD) 65-immunoreactive terminals was increased in the CA1 area when compared with control slices. Treatment with the glutamate receptor antagonist 6,7dinitroquinoxaline-2,3-dione decreased the density of GAD65immunoreactive terminals in the stratum oriens of CA1. These treatments had parallel effects on the density of GABAimmunoreactive processes. Electron microscopic analysis after
\end{abstract}

postembedding immunogold labeling with antibodies against GABA indicated that bicuculline treatment increased the density of inhibitory but not excitatory synapses. Application of exogenous BDNF partly mimicked the stimulatory effect of bicuculline on GAD65-immunoreactive terminals. Finally, antibodies against BDNF, but not antibodies against nerve growth factor, decrease the density of GAD65-immunoreactive terminals in bicucullinetreated slices.

Thus, neuronal activity regulates the density of inhibitory synapses made by postnatal hippocampal interneurons, and BDNF could mediate part of this regulation. This regulation of the density of inhibitory synapses could represent a feedback mechanism aimed at maintaining an appropriate level of activity in the developing hippocampal networks.

Key words: rat; development; Ammon's horn; GABAergic neurons; interneurons; neurotrophins
Hippocampal interneurons inhibit pyramidal cells through the release of the neurotransmitter GABA (Freund and Buzsáki, 1996). According to their axonal projection and neurochemical characteristics, the interneurons can be classified into several subgroups (Freund and Buzsáki, 1996). Interneurons innervating the cell body of pyramidal neurons may exert the inhibitory effect by suppressing sodium-dependent action potentials, whereas interneurons innervating the dendrites of pyramidal neurons may suppress calciumdependent dendritic spikes (Miles et al., 1996).

There is an important maturation of hippocampal excitatory transmission during the postnatal period. Non-NMDA glutamatergic transmission becomes prominent at the end of the first postnatal week, simultaneous with the establishment of the hyperpolarizing effects of GABA (Ben-Ari et al., 1989, 1997; Hosokawa et al., 1994; Durand et al., 1996; Petralia et al., 1999). The first postnatal month is also characterized by an increase in the number of excitatory synapses (Steward and Falk, 1991). Thus, the number and/or efficiency of inhibitory synapses may also increase during the postnatal period to adjust the strength of inhibition to counter the increased number of excitatory synapses.

Neuronal activity is a good candidate to regulate the development of inhibitory synapses. After chronic blockade of neuronal

Received March 2, 2000; revised Aug. 8, 2000; accepted Aug. 14, 2000.

This work was supported by Institut National de la Santé et de la Recherche Médicale Grant U106. C.S. and S.M. are Centre National de la Recherche Scientifique investigators. We thank Denis Le Cren for photographic assistance. We are grateful to Dr. Yves-Alain Barde for providing antibodies against NGF and to Dr. Kristy Ilinsky for providing antibodies against GAD65. We thank Dr. Fredrick Seil for advice regarding the experiments with blocking antibodies. We also thank Dr. Benedikt Berninger, Dr. Jonathan Cooper, Dr. Isabelle Dusart, and Dr. Patricia Gaspar for critical reading of this manuscript.

Correspondence should be addressed to S. Marty, Institut National de la Santé et de la Recherche Médicale U106, Hôpital de la Salpêtrière, Pavillon de l'Enfance et de l'Adolescence, 47 Boulevard de l'Hôpital, 75651 Paris cedex 13, France. E-mail: marty@chups.jussieu.fr.

Copyright (C) 2000 Society for Neuroscience $0270-6474 / 00 / 208087-09 \$ 15.00 / 0$ activity in cortical cell cultures, the transfer to control medium results in an increased activity likely attributable to, at least in part, a decrease of GABA-mediated inhibition (Rutherford et al., 1997). The neurotrophin brain-derived neurotrophic factor (BDNF) might mediate this activity-dependent modulation of synaptic inhibition. BDNF is synthesized and released by pyramidal neurons in an activity-dependent manner (Thoenen, 1995). BDNF treatment prevents the decrease of GABA-mediated inhibition during chronic blockade of neuronal activity (Rutherford et al., 1997). Furthermore, downregulation of BDNF in hippocampal cultures reduces the frequency of miniature IPSCs (Murphy et al., 1998). However, these experiments do not discriminate between the effects of neuronal activity and BDNF on either the efficacy or the number of inhibitory synapses. The hypothesis of a presumptive control of the number of inhibitory synapses by neuronal activity has been evaluated previously in ultrastructural studies. For instance, in organotypic cerebellar cultures, neuronal activity exerts its effects on synaptic inhibition by increasing the number of inhibitory synapses (Seil et al., 1994), and this regulation seems to be mediated by BDNF (Seil, 1999). Control of the number of inhibitory synapses by neuronal activity also occurs in the somatosensory cortex in vivo, because sensory deprivation during development causes a specific decrease in the number of GABAergic synapses in the rat barrel field cortex (Micheva and Beaulieu, 1995).

The present study was undertaken to determine whether neuronal activity and BDNF can regulate the density of inhibitory synapses made by postnatal hippocampal interneurons. Organotypic slice cultures of the hippocampus were used, because both excitatory transmission and BDNF expression mature in the slices with a similar time course to that seen in vivo (Buchs et al., 1993; Förster et al., 1993; Muller et al., 1993). Slices were taken from 7-d-old rats, when GABAergic transmission becomes hyperpolarizing and non-NMDA glutamatergic transmission is established (Ben-Ari et al., 1997). Endogenous neuronal activity was manipu- 
lated by chronic application of antagonists of either $\mathrm{GABA}_{\mathrm{A}}$ or non-NMDA glutamate receptors. The effects of these treatments on GABAergic synapses were evaluated with light microscopy, using antibodies against GABA or the GABA-synthesizing enzyme glutamic acid decarboxylase (GAD) 65 and ultrastructurally with the postembedding immunogold labeling with antibodies against GABA. The quantitative results indicate that neuronal activity regulates the density of inhibitory synapses made by postnatal hippocampal interneurons and that BDNF could mediate part of this process.

\section{MATERIALS AND METHODS}

Slice culture. Hippocampal slice cultures were prepared according to the method developed by Stoppini et al. (1991), except that a defined medium was used. Seven-day-old Wistar rats (Iffa Credo, L'arbresle, France) were decapitated, and their brains were rapidly removed. Hippocampi were dissected in Gey's balanced salt solution (catalog \#24260-028; Life Technologies, Cergy Pontoise, France) with $5 \mathrm{mg} / \mathrm{ml}$ glucose under sterile conditions. Slices, $350-\mu \mathrm{m}$-thick, were cut perpendicular to the septotemporal axis of the hippocampus using a McIllwain tissue chopper (Mickle Laboratory, Surrey, UK). Hippocampal slices were first transferred into the culture medium, separated, and ultimately transferred onto Millicell-CM membranes (Millipore, St. Quentin Yvelines, France). Twelve adjacent slices were obtained per brain. Adjacent slices were transferred onto different Millicells to compare the effects of the treatments with adjacent control material.

The Millicell membranes were kept above $750 \mu \mathrm{l}$ of defined medium in six-well plates. The medium consisted of minimum essential medium (catalog \#11012-010; Life Technologies), 1\% D-glucose, $5 \mathrm{~mm}$ Tris-HCl, $100 \mu \mathrm{g} / \mathrm{ml}$ bovine serum albumin (BSA), $100 \mu \mathrm{g} / \mathrm{ml}$ transferrin, $16 \mathrm{ng} / \mathrm{ml}$ putrescin, $40 \mathrm{ng} / \mathrm{ml} \mathrm{Na}$-selenium, $30 \mathrm{ng} / \mathrm{ml}$ tri-iodothyronin, $5 \mu \mathrm{g} / \mathrm{ml}$ insulin, and $60 \mathrm{ng} / \mathrm{ml}$ progesterone. All chemicals were purchased from Sigma (St. Quentin Fallavier, France). Slices were incubated at $35^{\circ} \mathrm{C}$ in $5 \% \mathrm{CO}_{2}$. The medium was exchanged every second or third day.

Pharmacological treatments. To manipulate neuronal activity in the slices, either $10 \mu \mathrm{M}$ of the GABA receptor antagonist bicuculline (Sigma) or $20 \mu \mathrm{M}$ of the non-NMDA glutamate receptor antagonist 6,7dinitroquinoxaline-2,3-dione (DNQX) (Sigma) were added to the medium during the $13 \mathrm{~d}$ of the cultivation period.

One microliter of recombinant human BDNF (Chemicon, Temecula, $\mathrm{CA}$ ) at a dilution of $100 \mathrm{ng} / \mu \mathrm{l}$ in PBS (catalog \#14200-067; Life Technologies) containing $0.1 \%$ BSA (Sigma) was applied directly on top of each slice as described previously (Marty et al., 1996). The neurotrophin was applied twice to the slices at 7 and 10 DIV, after exchange of the medium. Recombinant human neurotrophin-3 (NT-3) (Upstate Biotechnology, Lake Placid, NY) at a dilution of $100 \mathrm{ng} / \mu \mathrm{l}$ in PBS containing $0.1 \%$ BSA was applied as described above for BDNF. The neurotrophins were applied to untreated or DNQX-treated slices. In this last case, adjacent slices treated with DNQX only were used as controls. One microliter of PBS containing $0.1 \%$ BSA was applied to control slices as described above for the neurotrophins.

Antibodies against BDNF $(50 \mu \mathrm{g} / \mathrm{ml}$ ) (which is considered as specific by the provider; catalog \#G1641; Promega, Madison, WI) or antibodies against nerve growth factor (NGF) [a gift of Dr. Yves-Alain Barde (MaxPlanck-Institute, Martinsried, Germany)] were added to the medium during the $13 \mathrm{~d}$ in culture. The medium containing the antibodies was exchanged every second or third day. The antibodies were added to bicuculline-treated slices, and adjacent slices treated with bicuculline alone were used as controls.

Immunohistochemistry for light microscopy. After $13 \mathrm{~d}$ in culture, control and adjacent treated slices were fixed for $1.5 \mathrm{hr}$ in $4 \%$ paraformaldehyde in phosphate buffer $\left(0.12 \mathrm{M}\right.$ PBS, pH 7.4) at $4^{\circ} \mathrm{C}$, rinsed several times in PBS, and incubated for $1 \mathrm{hr}$ in $0.12 \mathrm{M}$ phosphate buffer, $\mathrm{pH} 7.4$, containing $0.9 \%$ $\mathrm{NaCl}, 0.25 \%$ Triton $\mathrm{X}-100,0.1 \%$ gelatin, $0.1 \%$ sodium azide (PBSGTA), and lysine $(0.1 \mathrm{M})$. The slices were then incubated overnight with antibodies raised against GAD65 [1:1000; GAD-6, this monoclonal antibody developed by D. I. Gottlieb (Washington University School of Medicine, St. Louis, MO) was obtained from the Developmental Studies Hybridoma Bank developed under the auspices of the National Institute of Child Health and Human Development and maintained by the University of Iowa, Department of Biological Sciences (Iowa City, IA)] or GABA (1:2000; rabbit polyclonal antibodies A-2052; Sigma) diluted in PBSGTA. After washes, the slices were incubated for $3 \mathrm{hr}$ with a solution containing either goat anti-mouse or goat anti-rabbit CY3 (1:1000; Jackson ImmunoResearch, West Grove, PA). After washes, the slices were mounted in mowiol (Calbiochem, La Jolla, CA), examined, and photographed with a Zeiss (Oberkochen, Germany) Axiophot.

Quantification of the density of GAD65-immunoreactive puncta and $G A B A$-immunoreactive processes in the CA1 area. Immunofluorescence was observed with a Zeiss LSM 410 confocal microscope, using $543 \mathrm{~nm}$ excitation wavelength and $570 \mathrm{~nm}$ emission wavelength. The explants were observed with the $63 \times / 1.4$ numerical aperture lens (Plan-Apochromat; Zeiss). The pinhole was set at 20 , and the resolution was $0.75 \mu \mathrm{m}$ on $z$-axis Confocal sections of the labeling were obtained every $1 \mu \mathrm{m}$ in the middle of the hippocampal slices in the CA1 region. The labeling was present in $10-15 \mu \mathrm{m}$ in the thickness of the slices. For GAD65 immunoreactivity, three areas were sampled, in the stratum oriens, the stratum pyramidale, and the stratum radiatum. For GABA immunoreactivity, only the stratum oriens was sampled because of the poor quality of labeling in the other areas of the CA1 subfield. Three-dimensional reconstructions of these confocal sections were then obtained using LSM software (Zeiss) and printed at a magnification of $880 \times$.

For quantification of the density of GAD65-immunoreactive puncta or of GABA-immunoreactive processes, a $36 \mathrm{~cm}^{2}$ square with a lattice of puncta spaced by $0.5 \mathrm{~cm}$ was applied over the prints of the threedimensional reconstructions. The number of points of the lattice hitting the elements of interest was then counted to determine the density of these elements (Gundersen et al., 1988). For each treatment, counts were performed blindly in slices taken from three to five animals. For each animal, two or three slices were used as control, and two or three adjacent slices were treated. At least two independent experiments were done for each experimental condition. The mean of the values was calculated for each animal for either the control or the treated slices. The mean value, SD, and SEM were then calculated from these mean values obtained with different animals. Because we were interested in the effects of the treatments relative to controls rather than in absolute values, these mean values are presented as percentages of control values, together with the SEM. The number of animals for each condition is indicated below each bar of the histograms in the figures. Comparison between the mean values of control and treated slices was performed by statistical analysis using the two-tailed unpaired Student's $t$ test.

Postembedding immunohistochemistry. After 13 DIV, the culture medium of control and treated slices was exchanged for a solution containing $3 / 4 \mathrm{vol}$ of culture medium $-1 / 4 \mathrm{vol}$ of fixative $(1 \%$ paraformaldehyde and $2.5 \%$ glutaraldehyde in PBS) for $5 \mathrm{~min}$, followed by $1 / 2$ vol of culture medium $-1 / 2$ vol of fixative for $5 \mathrm{~min}$, followed by $1 / 4 \mathrm{vol}$ of culture medium$3 / 4$ vol of fixative for $5 \mathrm{~min}$, followed by fixative. Then the fixative was added also over the slices for $2 \mathrm{hr}$ at $4^{\circ} \mathrm{C}$. The slices were then transferred into a solution containing $2 \%$ osmium tetroxide for $2 \mathrm{hr}$. After washes, the slices were stained en bloc with a solution containing $2 \%$ uranyl acetate for 45 $\mathrm{min}$. After washes, the slices were dehydrated in graded ethanols and flat embedded in Araldite. For each slice, one block containing the CA1 area was trimmed out from the middle of the slice. These blocks were sectioned with a Reichert-Jung ultramicrotome. In preliminary experiments, $1-\mu \mathrm{m}$ thick semithin sections were made in 26 control, 10 bicuculline-treated, and 12 DNQX-treated slices. One every 6th, 10th, or 15th section was stained with $0.5 \%$ toluidine blue (catalog \#1.15930; Merck, Darmstadt, Germany) in 1\% di-sodium tetraborate (catalog \#6306; Merck) in distilled water. These preliminary experiments indicated that areas of necrosis were not observed in our preparation, that the final thickness of the slices was $\sim 180$ $\mu \mathrm{m}$, and that there were no significant differences between the thickness of control and bicuculline- or DNQX-treated slices. Ultrathin sections were collected onto 300 mesh nickel grids at a depth of $90 \mu \mathrm{m}$ in the thickness of control or bicuculline-treated slices.

The ultrathin sections were treated for 5 min with $1 \%$ periodic acid, washed with ultrapure water, treated with $1 \%$ sodium metaperiodate, washed, and incubated 45 min with $5 \%$ bovine serum albumin in Trisbuffered saline, $\mathrm{pH}$ 7.6. After washes, the sections were incubated overnight at $4^{\circ} \mathrm{C}$ with rabbit polyclonal antibodies against GABA (1:250; catalog \#0602; Immunotech, Marseille, France). After washes, the sections were incubated with goat anti-rabbit immunogold conjugate with $15 \mathrm{~nm}$ gold particles (1:10; EM-GAR15; British Bio Cell International, Cardiff, UK) for $1 \mathrm{hr}$. After rapid washes in ultrapure water, the sections were stained with uranyl acetate and lead citrate and examined with a Philips CM100.

Quantification of the density and morphological characteristics of GABAand non-GABA-immunoreactive synapses in the stratum oriens. Counts of GABA-immunoreactive synapses were performed on sections taken from four animals, with two or three control and two or three adjacent bicuculline-treated slices analyzed per animal. For each slice, only one section was analyzed. For each section, two squares of the grid located in the stratum oriens were systematically screened, each square measuring $3969 \mu \mathrm{m}^{2}$, and the GABAergic synapses that had their synaptic junction in the plane of the section were counted. Stereological methods were not used for counting the synapses, because our quantification was not intended to obtain absolute values for which other counting methods are more appropriate (Guillery and Herrup, 1997). Instead, our counts were determined solely to compare relative synaptic densities in treated and control slices. GABAergic presynaptic terminals contained synaptic vesicles and gold particles and could therefore easily be identified. The synaptic junctions were identified by the slight widening of the synaptic cleft and the parallel disposition of presynaptic and postsynaptic membranes undercoated by cytoplasmic differentiations (Peters et al., 1991). In three control and three adjacent treated sections, the counts were repeated blindly. The GABAergic synapses were also photographed at a $27,500 \times$ magnification on sections taken from three of the animals used for quantification of synaptic number, with three control and three adjacent bicuculline-treated slices per animal. The electron photomicrographs were printed at a magnification of $60,000 \times$. Using these photomicrographs, the area of the presynaptic terminals and the length of the synaptic junctions were measured, and the number of gold particles was counted. The gold particles in mitochondria 

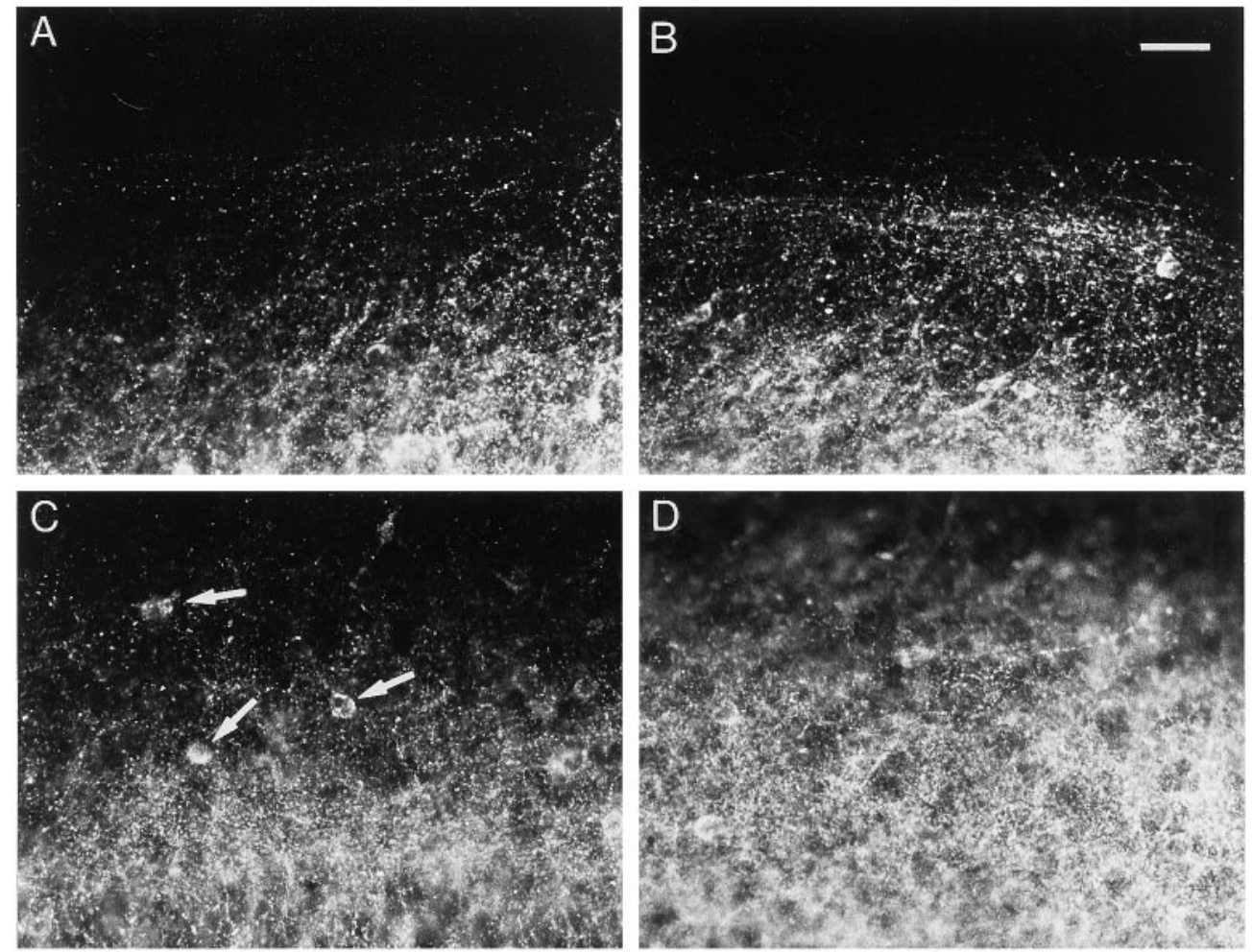

Figure 1. Effects of bicuculline treatment on GAD65 immunoreactivity. $A$, $C, E$, Control slice. $B, D, F$, Bicucullinetreated slice. $A, B$, Stratum oriens at the border of the slice. $C, D$, Middle of the stratum oriens. $E, F$, Stratum pyramidale $(s p)$ with part of the stratum oriens (so) and part of the stratum radiatum $(s r)$. Arrows point to neuronal cell bodies. Note the increased density of GAD65-immunoreactive puncta after bicuculline treatment, which is particularly evident in the stratum oriens. Scale bar, $40 \mu \mathrm{m}$.
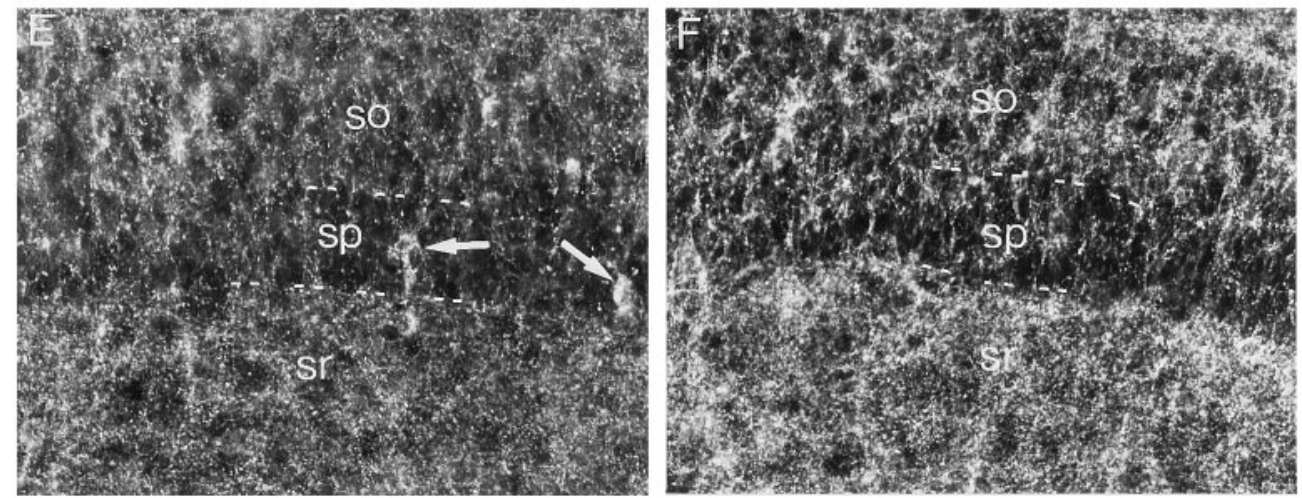

were excluded. The area of presynaptic terminals was measured by applying a lattice of puncta spaced by $1 \mathrm{~cm}$ over the photographs. The number of points overlying presynaptic terminals was counted to determine the area of these terminals (Gundersen et al., 1988).

Counts of non-GABA-immunoreactive synapses were performed on sections taken from three animals, with three control and three adjacent bicuculline-treated slices analyzed per animal. The counts of non-GABAimmunoreactive synapses were performed blindly in the squares in which GABAergic synapses were previously counted. Non-GABAergic synapses with a presynaptic terminal containing at least three synaptic vesicles were counted. These non-GABAergic synapses exhibited a widened synaptic cleft and thicker postsynaptic differentiations than the GABA-labeled synapses, and they most probably belong to excitatory synapses (Peters et al., 1991). An analysis of 84 presynaptic terminals from this type of synapses indicated that they contained a very low number of gold particles, with a mean value of 0.1 particles $/ 0.03 \mu \mathrm{m}^{2}$ in control slices and 0.07 particles $/ 0.03 \mu \mathrm{m}^{2}$ in bicuculline-treated slices.

Results were obtained in two independent experiments. The distribution of the values from slices of each animal, for each of the parameters that were analyzed, i.e., area of GABAergic presynaptic terminals, length of synaptic junctions of GABAergic synapses, and density of gold particles in GABAergic presynaptic terminals, exhibited one clear peak. Furthermore, the distribution of these values was similar in control and bicucullinetreated slices. The mean of the values was calculated for each animal for either control or treated sections. The mean value, SD, and SEM were then calculated from these mean values obtained with different animals. Because we were interested in the effects of the treatments relative to control rather than in absolute values, these mean values were presented as percentages of control values, together with the SEM. The number of animals is indicated below each bar of the histograms in the figures. Comparisons between the mean values of control and treated slices were performed by statistical analysis using two-tailed, unpaired Student's $t$ test.

\section{RESULTS}

Flattening of hippocampal slices was apparent after $2 \mathrm{~d}$ in culture. This flattening increased progressively over time, particularly at the edge of the slices. Nevertheless, the slices retained their structural organization over the incubation period. The pyramidal and dentate granule cell layers remained easily detectable, except for the infra-pyramidal blade of the dentate gyrus, which is generated at a later date and could not be recognized clearly as a cell layer. Treatment of the slices with bicuculline, DNQX, BDNF, or NT-3 did not induce conspicuous changes either in the size of the slices or their organotypic organization. We selected the CA1 area to examine the effects of modifications of neuronal activity or neurotrophin levels on GAD65 immunoreactivity, GABA immunoreactivity, or synapse density.

\section{Effects on GAD65 immunoreactivity of treatments with $\mathrm{GABA}_{\mathrm{A}}$ or non-NMDA glutamate receptor antagonists, BDNF or NT-3, or antibodies against BDNF or NGF}

After 13 DIV, a dense, punctate GAD65 immunolabeling was observed throughout the CA1 area (Fig. $1 A, C, E$ ). This punctate immunostaining was less dense in the stratum pyramidale than in the other layers (Fig. 1E). Scattered GAD65-immunoreactive soma were occasionally observed (Fig. $1 C, E$ ).

In bicuculline-treated slices, an increase in the density of GAD65-immunoreactive puncta was evident in all layers (Fig. 

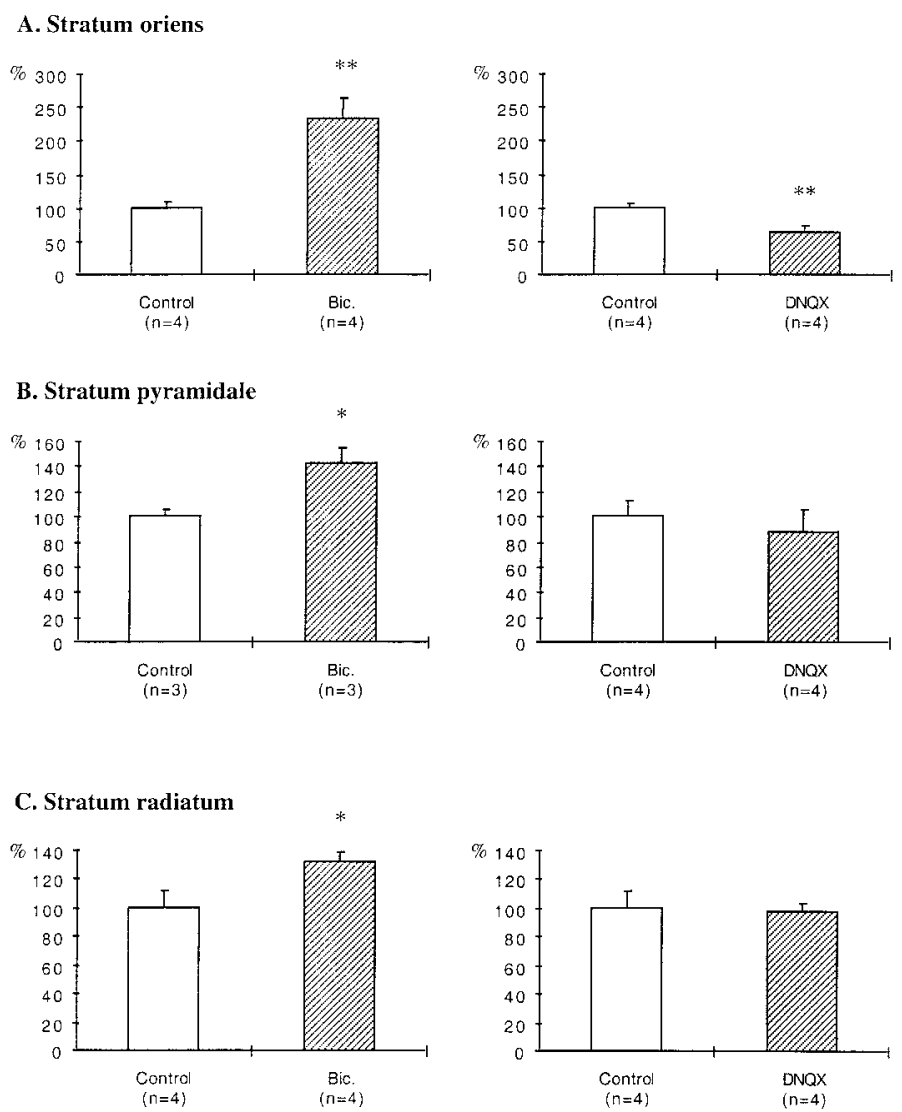

Figure 2. Histograms illustrating the effects of bicuculline and DNQX treatments on the density of GAD65-immunoreactive puncta. Results are presented as percentages of controls (white bars). $n$, Number of animals; Bic., bicuculline. ${ }^{*} p<0.05 ;{ }^{* *} p<0.01$.

$1 B, D, F)$, although it was more prominent in the stratum oriens. Quantification of the density of GAD65-immunoreactive puncta indicated a $133 \%$ increase in the stratum oriens, a $42 \%$ increase in the stratum pyramidale, and a $32 \%$ increase in the stratum radiatum (Fig. $2 A-C$ ).

In DNQX-treated slices, a decrease in the density of GAD65immunoreactive puncta was observed in the stratum oriens (Fig. $3 A-D)$. Quantification of this density revealed a $38 \%$ decrease in the stratum oriens, whereas no significant decreases were observed in the stratum pyramidale or in the stratum radiatum (Fig. $2 A-C$ ).

In slices treated with BDNF during the last 6 DIV, the density of GAD65-immunoreactive puncta increased in the stratum oriens. Quantification of the density of these puncta indicated a $63 \%$ increase in the stratum oriens (Fig. $4 A$ ). BDNF has been shown to facilitate excitatory transmission (Berninger and Poo, 1996; Schuman, 1999). To exclude the possibility that the effects of BDNF on the density of GAD65-immunoreactive puncta could arise from a direct increase in excitatory transmission, BDNF was applied to DNQX-treated slices. Under these conditions, the density of GAD65-immunoreactive puncta was also increased in the stratum oriens $(43 \%)$ when compared with the stratum oriens of DNQXtreated slices that received BSA only (Fig. 4B). However, stimulation with NT-3 did not affect the density of GAD65immunoreactive puncta in the stratum oriens of DNQX-treated slices (Fig. 4C).

In bicuculline-treated slices that received in addition antibodies against BDNF, the density of GAD65-immunoreactive puncta was decreased in the stratum oriens when compared with adjacent slices treated only with bicuculline. Quantification of the density of these puncta indicated a $38 \%$ decrease in the stratum oriens (Fig. $4 D$ ). On the other hand, addition of antibodies against NGF did not affect the density of GAD65-immunoreactive puncta in the stratum oriens of bicuculline-treated slices (Fig. 4E).

\section{Effects of treatments with $\mathrm{GABA}_{\mathrm{A}}$ and non-NMDA glutamate receptor antagonists on GABA immunoreactivity}

In cultures stained with anti-GABA antibodies, immunoreactivity was very dense and not restricted to punctated elements. In addition, despite the use of confocal microscopy, immunostained structures were sharply defined only in the thinnest regions, i.e., at the borders of the slices, containing the stratum oriens (Fig. 5). In thicker slice regions, containing the strata pyramidale and radiatum, the immunolabeling was somewhat blurred and variable, probably because of the large amounts of GABA present in the thick part of the slices and its presumed leakage during fixation. As such, quantitative analyses were therefore restricted to the stratum oriens.

In control slices after 13 DIV, a dense network of labeled processes was observed in the stratum oriens (Fig. $5 A, C$ ). At the periphery of the slices, the network consisted of a bundle of thin, varicose, parallel processes (Fig. $5 A$ ), whereas such thin, varicose processes ran in every direction in the middle of the stratum oriens (Fig. 5C). Other labeled processes were also distinguished, which were thicker and tapered along their course (Fig. 5A). Faintly stained cell bodies were also observed (Fig. 5A,C).

In bicuculline-treated slices, an overall increase in the density of GABAergic processes occurred in both the peripheral parallel bundle and the middle of the stratum oriens (Fig. $5 B, D$ ). There was no increase in the staining intensity of cell bodies (Fig. $5 C, D$ ). Quantification of the density of GABA-immunoreactive processes indicated a $29 \%$ increase in the stratum oriens (Fig. 6A).

In DNQX-treated slices, a decrease in the density of GABAergic processes was observed both at the border and in the middle of the stratum oriens (Fig. $7 A-D$ ). This decrease coexisted with an increase in the intensity of immunostaining of thick processes and cell bodies (Fig. 7B,D). Quantification of the density of GABAimmunoreactive processes indicated a $31 \%$ decrease in the stratum oriens (Fig. 6B).

\section{Effects of treatment with the $\mathrm{GABA}_{\mathrm{A}}$ receptor antagonist bicuculline on GABAergic and non- GABAergic synapses in the stratum oriens}

The ultrastructural study allowed us to ascertain that neurons in the slices were healthy and that there were no signs of degeneration in synaptic terminals. In control slices after 13 DIV, the GABAergic axon terminals in the stratum oriens were readily identified on the basis of containing both synaptic vesicles and gold particles, together with the symmetric appearance of their synaptic complexes (Fig. 8A). The lower values found in the 104 GABAergic synapses analyzed were five vesicles and seven gold particles (mean value, 42 gold particles per terminal; the gold particles in mitochondria were not counted). The shape of these axon terminals was most often irregular, varying from rounded to elongated. Sometimes, en passant terminals were observed. In bicuculline-treated slices, GABAergic synapses were also clearly identifiable in the stratum oriens (Fig. $8 B$ ). The lower values found in the 170 GABAergic synapses analyzed were seven vesicles and five gold particles (the gold particles in mitochondria were not counted). The density of GABAergic synapses in bicuculline-treated slices was increased by $79 \%$ when compared with adjacent, control slices (mean \pm SEM; $14 \pm 1$ GABAergic synapses per $7938 \mu \mathrm{m}^{2}$ in control slices, and $25 \pm 3$ GABAergic synapses per $7938 \mu \mathrm{m}^{2}$ in bicuculline-treated slices) (Fig. 9A).

Non-GABAergic synapses were distinguished from GABAergic synapses by their almost complete lack of gold particles (Fig. $8 A, B)$. Furthermore, the postsynaptic differentiations of nonGABAergic synapses were thicker and more electron-dense than those of GABAergic synapses (Fig. 8A,B). Non-GABAergic synapses were more frequently encountered than GABAergic synapses (mean \pm SEM; $135 \pm 8$ non-GABAergic synapses per 7938 $\mu \mathrm{m}^{2}$ ). However, bicuculline treatment did not significantly change the density of non-GABAergic synapses (mean \pm SEM; $114 \pm 18$ non-GABAergic synapses per $7938 \mu \mathrm{m}^{2}$ ) (Fig. 9B). 

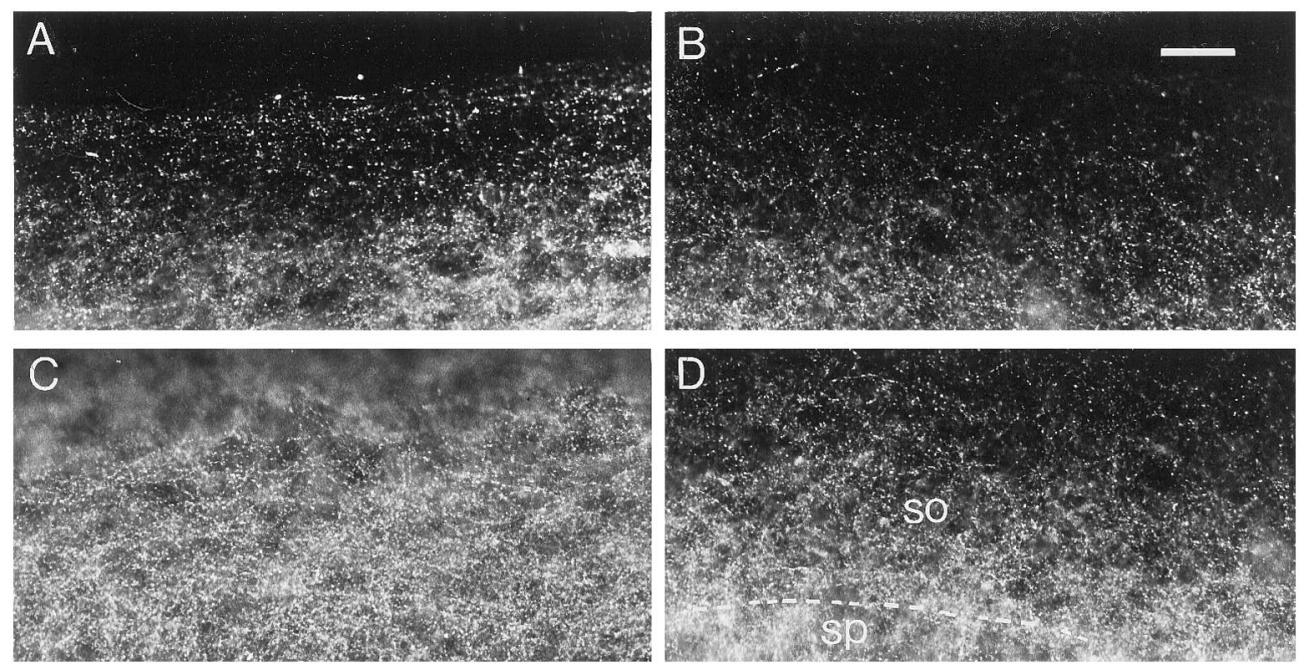

Figure 3. Effects of DNQX treatment on GAD65 immunoreactivity. $A, C$ Control slice. $B, D, \mathrm{DNQX}$-treated slice. $A, B$, Stratum oriens at the border of the slice. $C, D$, Middle of the stratum oriens. $s o$, Stratum oriens; $s p$, stratum pyramidale. Note the decreased density of GAD65-immunoreactive puncta in the stratum oriens after DNQX treatment. Scale bar, $40 \mu \mathrm{m}$.
In control slices, the mean area of GABAergic axon terminals was $0.5 \pm 0.03 \mu \mathrm{m}^{2}$, whereas the mean length of their synaptic complexes was $334 \pm 5 \mathrm{~nm}$, and the mean density of gold particles was 2.4 particles $/ 0.03 \mu \mathrm{m}^{2}$. The increase in the density of GABAergic synapses after bicuculline treatment occurred without detectable changes in the area of GABAergic terminals, the length of their synaptic complexes, or in the number of gold particles per unit area of terminal axoplasm (Fig. 9C-E).

\section{DISCUSSION}

The results of this study indicate that neuronal activity regulates the density (number per surface area) of GAD65-immunoreactive inhibitory terminals in organotypic slice cultures from rat postnatal hippocampus. When endogenous neuronal activity was increased by blocking $\mathrm{GABA}_{\mathrm{A}}$ receptors, the density of GAD65-immunoreactive terminals increased. An opposite effect was observed when neuronal activity was reduced by blockade of non-NMDA glutamate receptors. The density of GABA-immunoreactive processes was regulated in the same manner. Electron microscopic analysis indicated that neuronal activity exerted at least part of its effects by increasing the density of GABAergic synapses. BDNF application mimicked partly the effect of neuronal activity on GAD65immunoreactive terminals. Finally, antibodies against BDNF but not against NGF decreased the density of GAD65-immunoreactive terminals in bicuculline-treated slices. These results indicate that neuronal activity regulates the density of inhibitory synapses in organotypic slice cultures of postnatal hippocampus and that this process is partly mediated by BDNF.

\section{Activity-dependent regulation of the density of inhibitory synapses}

Subcellular fractionation and immunohistochemistry have revealed that GAD65 is enriched in presynaptic terminals (Erlander et al., 1991; Esclapez et al., 1994). Because $90 \%$ of hippocampal interneurons expressing GAD67 also express GAD65 (Stone et al., 1999), the latter is an optimal, light microscopic marker of presynaptic inhibitory terminals. In the present study, the density of GAD65-immunoreactive puncta was increased in response to an increased neuronal activity induced by bicuculline treatment, and, in the stratum oriens only, decreased in response to a decrease of neuronal activity after DNQX treatment. Changes in the density of GAD65-immunoreactive puncta did not result from differential shrinkage of the tissue, because the thickness of the slices remained constant after bicuculline or DNQX treatments (see Materials and Methods). These modifications were probably also independent from changes in the survival of inhibitory neurons, because spontaneous pyramidal cell death in hippocampal organotypic cultures can be blocked by glutamate receptor antagonists (Pozzo Miller et al., 1994). Thus, increasing neuronal activity should be expected to
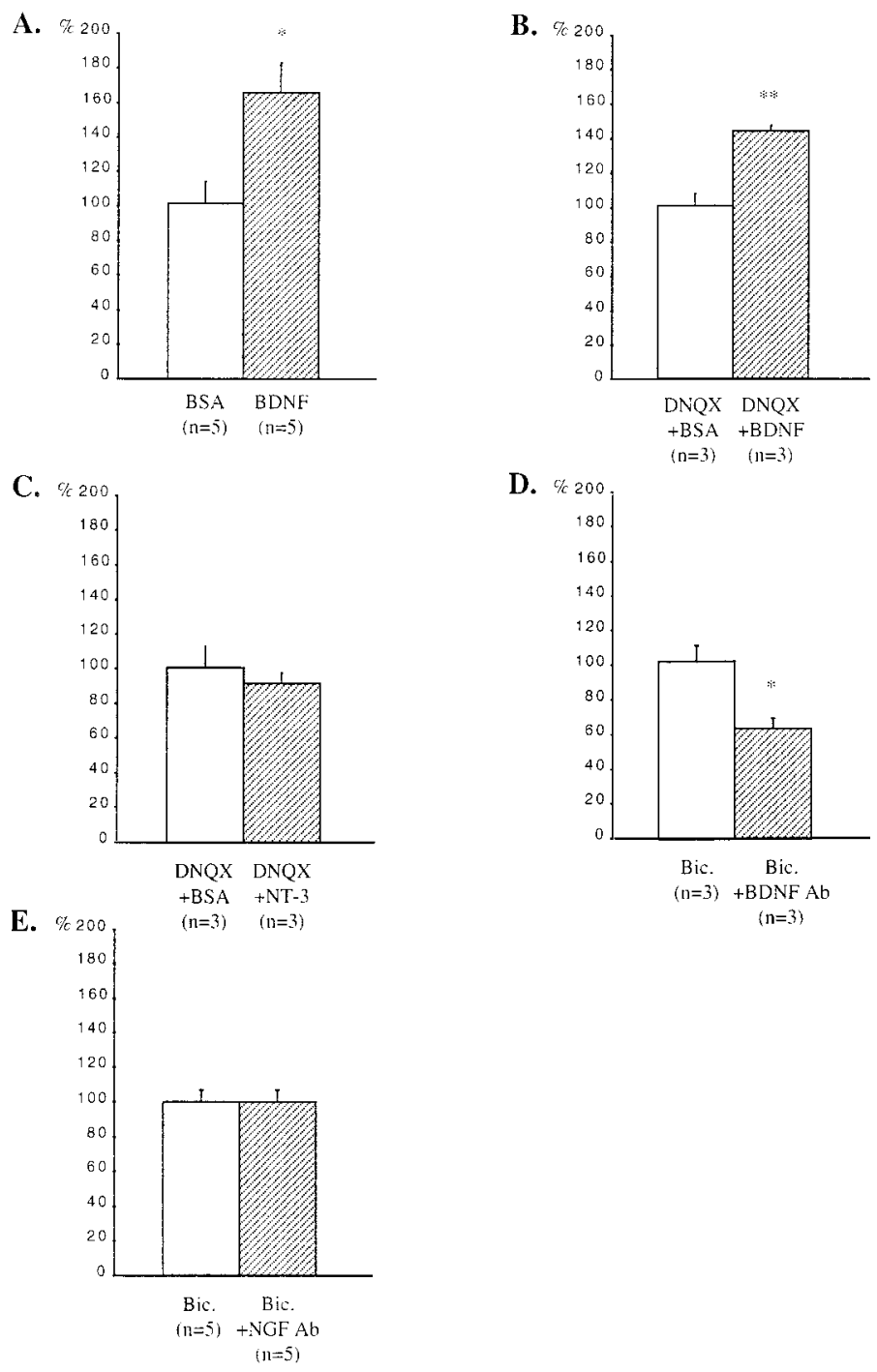

Figure 4. Quantification of the effects of treatments with BDNF or NT-3 and with antibodies against BDNF or NGF on the density of GAD65immunoreactive puncta in the stratum oriens. $A$, Untreated slices. $B, C$, DNQX-treated slices. $D, E$, Bicuculline-treated slices. Results are presented as percentages of controls (white bars). $n$, Number of animals; Bic., bicuculline. ${ }^{*} p<0.05 ;{ }^{*} p<0.01$. 

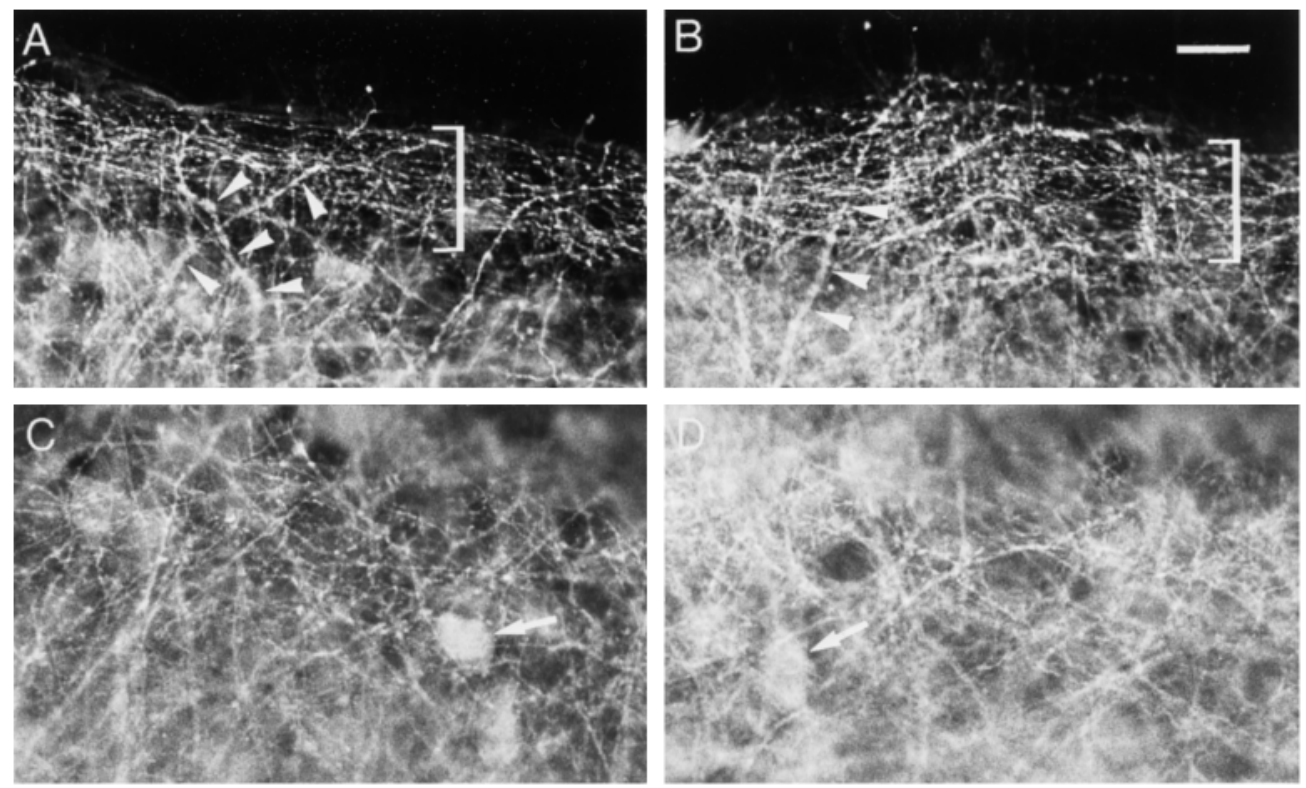

Figure 5. Effects of bicuculline treatment on GABA immunoreactivity. $A, C$, slice. $A, B$, Stratum oriens at the border of the slice. Brackets delineate a bundle of thin processes. Arrowheads point to thick processes. $C, D$, Middle of the stratum oriens. Arrows point to cell bodies. Note the increased density of GABAimmunoreactive processes after bicuculline treatment. Scale bar, $20 \mu \mathrm{m}$.

B.
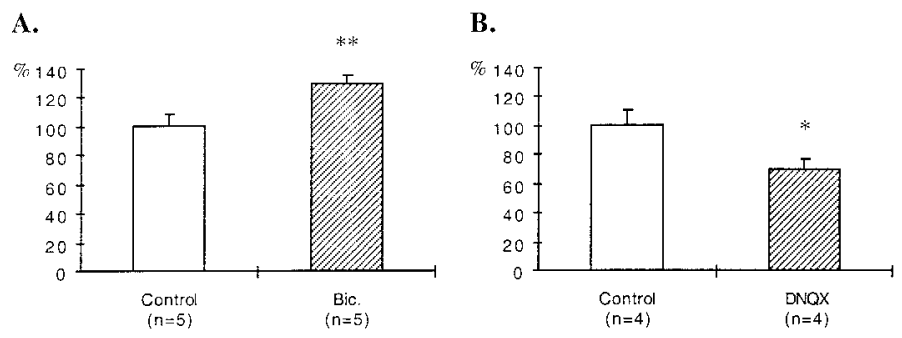

Figure 6. Quantification of the effects of bicuculline and DNQX treatments on the density of GABA-immunoreactive processes in the stratum oriens. Results are presented as percentages of controls (white bars). $n$, Number of animals; Bic., bicuculline. ${ }^{*} p<0.05 ;{ }^{* *} p<0.01$.

promote excitotoxic cell death rather than increasing the density of GAD65-immunoreactive puncta. Therefore, if the survival of interneurons is regulated by activity in the same manner as the survival of pyramidal neurons, the modulation of the density of GAD65-immunoreactive terminals most likely results from a modulation of the number of axon terminals per interneuron.

Different types of interneurons innervate the different layers of the hippocampal slices (Freund and Buzsáki, 1996). After bicuculline treatment, GAD65-immunoreactive terminals increased their overall density, suggesting that neuronal activity controls the density of GAD65-immunoreactive terminals of various types of interneurons. In contrast, DNQX treatment decreased the density of GAD65-immunoreactive terminals only in the stratum oriens. Interneurons that exclusively innervate the stratum oriens (Parra et al., 1998) could be particularly sensitive to activity deprivation. Alternately, the effects of modifications of neuronal activity may be more important for axon terminals in the stratum oriens, even for interneurons that also have axon terminals in other layers. This more valid hypothesis is supported by our observation that, after bicuculline treatment, despite the general numerical increase of GAD65-labeled terminal in different layers of the CA1 area, the increase in the stratum oriens was the most prominent. Whatever the reason for this different response in specific regions, the regulation of the density of GAD65-immunoreactive terminals is less profound after DNQX than after bicuculline treatment, suggesting that this regulation is more sensitive to an increase than to a decrease in neuronal activity.

The modulation of the density of GAD65-immunoreactive puncta by neuronal activity suggests an effect of the activity on the real number of inhibitory synapses, providing some indirect evi- dence on the role of neuronal activity in the establishment of hippocampal circuitry. However, the observed changes could be also explained by an activity-dependent regulation of GAD65 mRNA transcripts, thereby increasing GAD65 protein levels in already existing inhibitory terminals; more enzymatic protein would produce more biosynthesis of GABA. Such an hypothetical increase of both antigens contents could increase the density of axon terminals that contain GAD65 and GABA at levels above the threshold for immunohistochemical detection. Neither of these two mechanisms explains, however, the difference between the $133 \%$ increase in GAD65-positive terminals and the much lesser $(29 \%)$ increase in GABA-labeled processes observed in bicucullinetreated slices. This apparent discrepancy could be explained considering that GABA immunohistochemistry likely labels both dendrites and axons of interneurons. However, that an activitydependent modulation of the dendrites of the interneurons could contribute to the modifications of GABA immunoreactivity remains to be determined.

Our electron microscopy study does provide the required evidence in favor of an activity-dependent mechanism regulating the density of inhibitory synapses. The increase in numerical density found in the stratum oriens of cultures treated with bicuculline, occurred without the following: (1) changes in the surface area occupied by the terminals; (2) changes in the length of synaptic complexes; and (3) changes in the density of gold particles. Thus, it is likely that the observed numerical increase is neither the result of an enlargement of the terminals nor an increase of the GABA content. It can only be explained by a real augmentation in number of inhibitory synapses. The increase in the density of GABAimmunoreactive synapses after bicuculline treatment (79\%) was less important than the increase in the density of GAD65immunoreactive puncta $(133 \%)$. This numerical mismatch suggests that, in addition to regulating the density of GABAergic synapses, neuronal activity regulates also GAD65 levels, as reported in other systems (Hendry and Jones, 1988; Aamodt et al., 2000). The activity-dependent modulation of the density of inhibitory synapses during postnatal development could be a general process, because such a density is also regulated by neuronal activity in organotypic cerebellar cultures (Seil and Drake-Baumann, 1994; Seil et al., 1994) and in the rat barrel field neocortex in vivo (Micheva and Beaulieu, 1995). Finally, this modulation of the density of inhibitory synapses by neuronal activity could explain the decrease of GABA-mediated inhibition that follows activity blockade in neocortical cultures (Rutherford et al., 1997). 

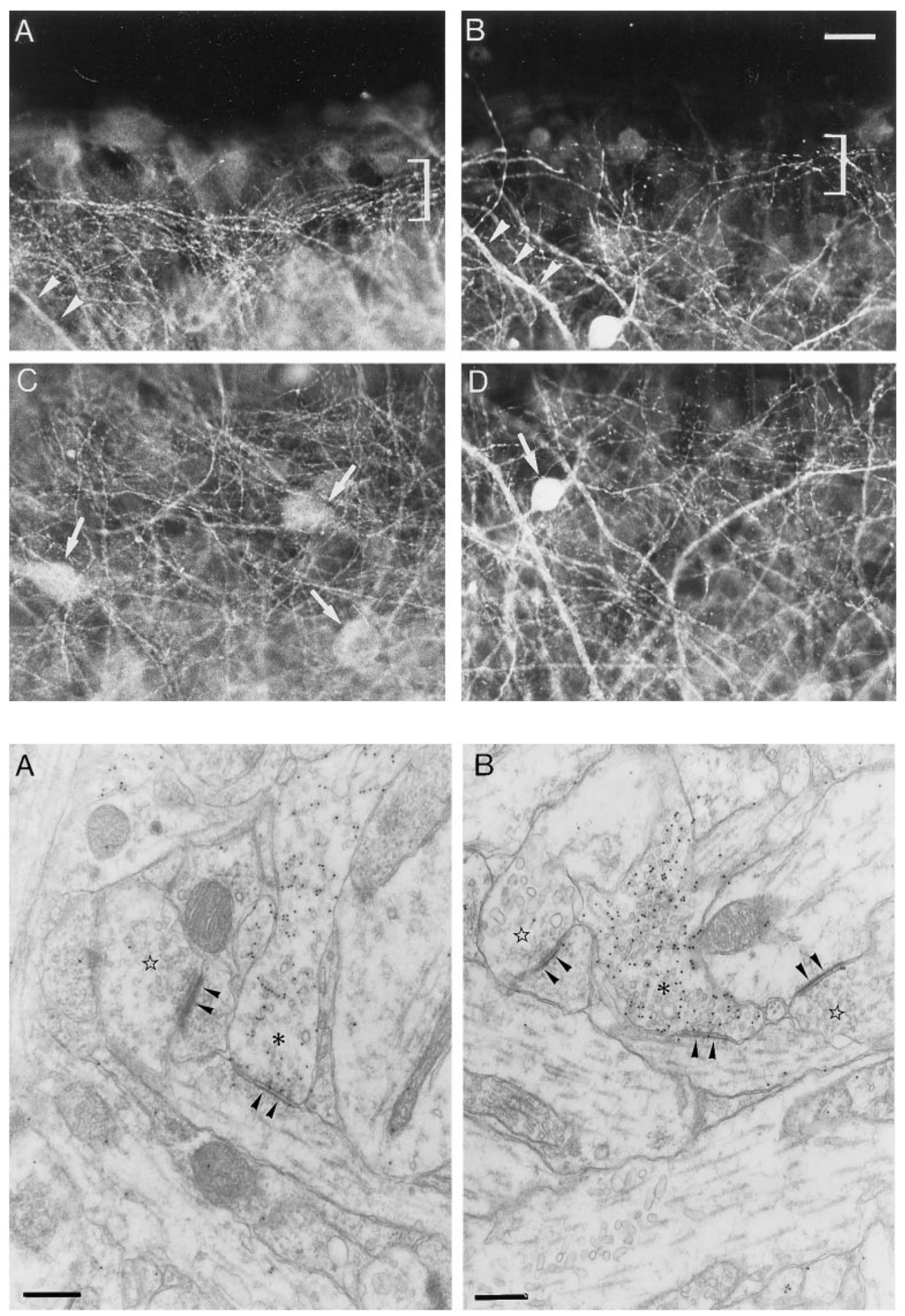

Figure 7. Effects of DNQX treatment on GABA immunoreactivity. $A, C$, Control slice. $B, D, \mathrm{DNQX}$-treated slice. $A$, $B$, Stratum oriens at the border of the slice. $C, D$, Middle of the stratum oriens. Arrowheads point to thick processes; arrows point to cell bodies; brackets delineate a bundle of thin processes. Note the decreased density of GABA-immunoreactive processes and the increased intensity of labeling of thick processes and cell body after DNQX treatment. Scale bar, $20 \mu \mathrm{m}$.

Figure 8. Electron photomicrographs of GABAergic synapses in control $(A)$ and bicuculline-treated $(B)$ slices. The asterisks mark labeled presynaptic terminals of GABAergic synapses, which contain pleomorphic synaptic vesicles, and establish type II synaptic complexes. Note the difference in the densities at the cytoplasmic faces of the postsynaptic differentiations (arrowheads) in GABAergic and non-GABAergic (stars) synapses. The latter correspond to type I synaptic complexes. Scale bars, $300 \mathrm{~nm}$.

\section{Possible involvement of BDNF in the activity-dependent regulation of the density of inhibitory synapses}

Neuronal activity regulates BDNF mRNA levels and BDNF release (Zafra et al., 1991, 1992; Goodman et al., 1996; Heymach et al., 1996; Canossa et al., 1997; Shieh et al., 1998; Tao et al., 1998; Mowla et al., 1999). BDNF treatment mimicked the effects of a raise in neuronal activity on the density of GAD65immunoreactive terminals. The effect of BDNF was specific, because NT-3 did not affect GAD65 immunoreactivity despite the fact that its receptor, TrkC, is expressed in the hippocampus (Barbacid, 1994). Furthermore, treatment with antibodies against BDNF decreased the density of GAD65-immunoreactive terminals in bicuculline-treated slices. This effect appeared specific, because antibodies against NGF did not affect GAD65 immunoreactivity, although NGF is synthesized and released by hippocampal neurons in an activity-dependent manner (Thoenen, 1995). These results are in agreement with previously published data showing that BDNF increases the number of axonal branches and the total length of GABA-immunoreactive axons in dissociated cultures from embryonic hippocampus (Vicario-Abejón et al., 1998). The effects of BDNF on inhibitory terminals are not limited to hippocampal neurons and has been also reported in organotypic cerebellar slices (Seil, 1999). Furthermore, BDNF is also implicated in the activity-dependent regulation of the development of ocular dominance in primary visual cortex in which it plays a key role in the modulation of intracortical inhibitory interneurons (Berardi and Maffei, 1999; Huang et al., 1999). However, treatment with antibodies against BDNF (the present study) did not fully 


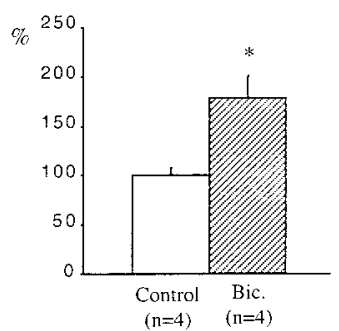

C. Area of GABAergic presynaptic terminals

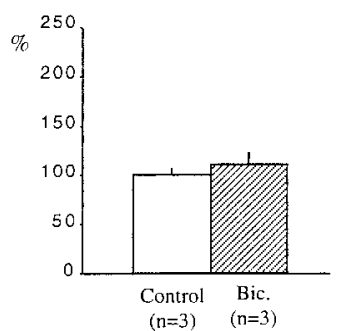

E. Density of gold particles in presynaptic terminals

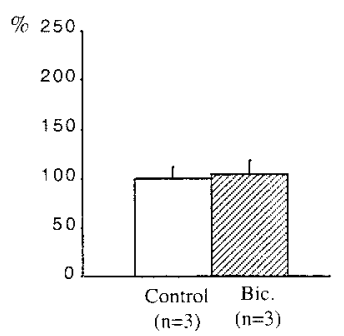

Figure 9. Quantification of the effects of bicuculline treatment on GABAergic and non-GABAergic synapses in the stratum oriens. Results are presented as percentages of controls (white bars). Bicuculline increased the density of GABAergic synapses $(A)$ but did not affect the density of non-GABAergic synapses $(B)$, the area of GABAergic presynaptic terminals $(C)$, the length of synaptic junctions of GABAergic synapses $(D)$, or the density of gold particles in GABAergic presynaptic terminals $(E) . n$, Number of animals; Bic., bicuculline. ${ }^{*} p<0.05$.

antagonize the effect of bicuculline on the numerical increase of GAD65-immunoreactive terminals. This partial effect could arise from insufficient blockade of available BDNF. Nevertheless, our observation that BDNF treatment reproduces only $47 \%$ of the bicuculline effect strongly suggests that this neurotrophin mediates only part of the effects of increased activity on inhibitory synapse density.

The mechanism of action of BDNF on inhibitory terminals remains to be elucidated. In this study, BDNF exerted its effect on GAD65-immunoreactive terminals in DNQX-treated slices. This result indicates that the increased density of GAD65immunoreactive terminals after BDNF treatment was not attributable to an enhancement of excitatory synaptic transmission by the neurotrophin (Berninger and Poo, 1996; Schuman, 1999). However, in addition to the activation of intracellular signaling pathways, BDNF may also depolarize neurons through the activation of a sodium ion conductance (Kafitz et al., 1999). Whether such depolarizing effects of BDNF contribute to the effects of this neurotrophin on inhibitory terminals remains to be tested.

From a functional point of view, the effect of BDNF on the density of inhibitory synapses may underlie the capability of chronic treatment with this neurotrophin to increase the frequency of inhibitory currents in dissociated cultures from neocortex or hippocampus (Rutherford et al., 1997; Murphy et al., 1998). Finally, such a regulation of inhibitory function by BDNF may occur in vivo, because BDNF overexpression accelerates the maturation of GABAergic inhibition in mouse visual cortex, in parallel with an acceleration of the maturation of GABAergic innervation (Huang et al., 1999).

\section{Possible functional consequences of the activity- dependent regulation of the density of inhibitory synapses}

The modifications of the density of inhibitory synapses occurred without concomitant changes of the density of excitatory synapses. This observation is in agreement with the finding that long-term treatment with a $\mathrm{GABA}_{\mathrm{A}}$ receptor blocker does not affect the number of dendritic spines in cultured hippocampal slices (Collin et al., 1997). The same specificity was also found in organotypic cerebellar cultures and in the rat barrel field cortex in vivo (Seil and Drake-Baumann, 1994; Micheva and Beaulieu, 1995). Such a regulation of the density of inhibitory synapses without changing the density of excitatory synapses may explain the imbalance of neuronal activity when cultures are returned to control medium after long-term blockade of excitatory or inhibitory activities. Cultures in which neuronal activity was blocked exhibit an increased activity (Furshpan and Potter, 1989; Segal and Furshpan, 1990; Corner and Ramakers, 1992; Seil and Drake-Baumann, 1994). In contrast, cultures in which neuronal activity was increased exhibit a decreased activity (Corner and Ramakers, 1992; Seil et al., 1994; Turrigiano et al., 1998). Such regulations may also take place in vivo. Increased spontaneous activity in the rat barrel field cortex after sensory deprivation during development may be related to a specific decrease in the number of GABAergic synapses (Micheva and Beaulieu, 1995). The specific modulation of the density of inhibitory synapses in response to changes in neuronal activity could therefore represent a feedback mechanism aimed at maintaining an appropriate level of activity in developing cortical networks.

\section{REFERENCES}

Aamodt SM, Shi J, Colonnese MT, Veras W, Constantine-Paton M (2000) Chronic NMDA exposure accelerates development of GABAergic inhibition in the superior colliculus. J Neurophysiol 83:1580-1591.

Barbacid M (1994) The Trk family of neurotrophin receptors. J Neurobiol 25:1386-1403.

Ben-Ari Y, Cherubini E, Corradetti R, Gaiarsa J-L (1989) Giant synaptic potentials in immature rat CA3 hippocampal neurones. J Physiol (Lond) 416:303-325.

Ben-Ari Y, Khazipov R, Leinekugel X, Caillard O, Gaiarsa J-L (1997) $\mathrm{GABA}_{\mathrm{A}}$, NMDA and AMPA receptors: a developmentally regulated "ménage à trois." Trends Neurosci 20:523-529.

Berardi N, Maffei L (1999) From visual experience to visual function: roles of neurotrophins. J Neurobiol 41:119-126.

Berninger B, Poo M-M (1996) Fast actions of neurotrophic factors. Curr Opin Neurobiol 6:324-330.

Buchs P-A, Stoppini L, Muller D (1993) Structural modifications associated with synaptic development in area CA1 of rat hippocampal organotypic cultures. Dev Brain Res 71:81-91.

Canossa M, Griesbeck O, Berninger B, Campana G, Kolbeck R, Thoenen H (1997) Neurotrophin release by neurotrophins: implications for activity-dependent neuronal plasticity. Proc Natl Acad Sci USA 94:13279-13286.

Collin C, Miyaguchi K, Segal M (1997) Dendritic spine density and LTP induction in cultured hippocampal slices. J Neurophysiol 77:1614-1623.

Corner MA, Ramakers GJA (1992) Spontaneous firing as an epigenetic factor in brain development: physiological consequences of chronic tetrodotoxin and picrotoxin exposure on cultured rat neocortex neurons. Dev Brain Res 65:57-64.

Durand GM, Kovalchuk Y, Konnerth A (1996) Long-term potentiation and functional synapse induction in developing hippocampus. Nature 381:71-75.

Erlander MG, Tillakaratne NJK, Feldblum S, Patel N, Tobin AJ (1991) Two genes encode distinct glutamate decarboxylases. Neuron 7:91-100.

Esclapez M, Tillakaratne NJK, Kaufman DL, Tobin AJ, Houser CR (1994) Comparative localization of two forms of glutamic acid decarboxylase and their mRNAs in rat brain supports the concept of functional differences between the forms. J Neurosci 14:1834-1855.

Förster E, Otten U, Frotscher M (1993) Developmental neurotrophin expression in slice cultures of rat hippocampus. Neurosci Lett 155:216-219. Freund TF, Buzsáki G (1996) Interneurons of the hippocampus. Hippocampus 6:345-470.

Furshpan EJ, Potter DD (1989) Seizure-like activity and cellular damage in rat hippocampal neurons in cell culture. Neuron 3:199-207. 
Goodman LJ, Valverde J, Lim F, Geschwind MD, Federoff HJ, Geller AI, Hefti F (1996) Regulated release and polarized localization of brainderived neurotrophic factor in hippocampal neurons. Mol Cell Neurosci 7:222-238

Guillery RW, Herrup K (1997) Quantification without pontification: choosing a method for counting objects in sectioned tissues. J Comp Neurol 386:2-7.

Gundersen HJ, Bendtsen TF, Korbo L, Marcussen N, Moller A, Nielsen K, Nyengaard JR, Pakkenberg B, Sorensen FB, Vesterby A, West MJ (1988) Some new, simple and efficient stereological methods and their use in pathological research and diagnosis. APMIS 96:379-394.

Hendry SHC, Jones EG (1988) Activity-dependent regulation of GABA expression in the visual cortex of adult monkeys. Neuron 1:701-712.

Heymach J, Krüttgen A, Suter U, Shooter EM (1996) The regulated secretion and vectorial targeting of neurotrophins in neuroendocrine and epithelial cells. J Biol Chem 271:25430-25437.

Hosokawa Y, Sciancalepore M, Stratta F, Martina M, Cherubini E (1994) Developmental changes in spontaneous GABA-A mediated synaptic events in rat hippocampal CA3 neurons. Eur J Neurosci 6:805-813.

Huang ZJ, Kirkwood A, Pizzorusso T, Porciatti V, Morales B, Bear MF, Maffei L, Tonegawa S (1999) BDNF regulates the maturation of inhibition and the critical period of plasticity in mouse visual cortex. Cell 98:739-755

Kafitz KW, Rose CR, Thoenen H, Konnerth A (1999) Neurotrophinevoked rapid excitation through TrkB receptors. Nature 401:918-921.

Marty S, Carroll P, Cellerino A, Castren E, Staiger V, Thoenen H, Lindholm D (1996) Brain-derived neurotrophic factor promotes the differentiation of various hippocampal non-pyramidal neurons, including Cajal-Retzius cells, in organotypic slice cultures. J Neurosci 16:675-687.

Micheva KD, Beaulieu C (1995) An anatomical substrate for experiencedependent plasticity of the rat barrel field cortex. Proc Natl Acad Sci USA 92:11834-11838.

Miles R, Toth K, Gulyás AI, Hajos N, Freund TF (1996) Differences between somatic and dendritic inhibition in the hippocampus. Neuron $16: 815-823$

Mowla SJ, Pareek S, Farhadi HF, Petrecca K, Fawcett JP, Seidah NG, Morris SJ, Sossin WS, Murphy RA (1999) Differential sorting of nerve growth factor and brain-derived neurotrophic factor in hippocampal neurons. J Neurosci 19:2069-2080.

Muller D, Buchs P-A, Stoppini L (1993) Time course of synaptic development in hippocampal organotypic cultures. Dev Brain Res 71:93-100.

Murphy DD, Cole NB, Segal M (1998) Brain-derived neurotrophic factor mediates estradiol-induced dendritic spine formation in hippocampal neurons. Proc Natl Acad Sci USA 95:11412-11417.

Parra P, Gulyás AI, Miles R (1998) How many subtypes of inhibitory cells in the hippocampus? Neuron 20:983-993.

Peters A, Palay SL, Webster HdeF (1991) The fine structure of the nervous system. New York: Oxford UP.

Petralia RS, Esteban JA, Wang Y-X, Partridge JG, Zhao H-M, Wenthold RJ, Malinow R (1999) Selective acquisition of AMPA receptors over postnatal development suggests a molecular basis for silent synapses. Nat Neurosci 2:31-36.

Pozzo Miller LD, Mahanty NK, Connor JA, Landis DMD (1994) Spontaneous pyramidal cell death in organotypic slice cultures from rat hippocampus is prevented by glutamate receptor antagonists. Neuroscience 63:471-487.

Rutherford LC, DaWan A, Lauer HM, Turrigiano GG (1997) Brainderived neurotrophic factor mediates the activity-dependent regulation of inhibition in neocortical cultures. J Neurosci 17:4527-4535.

Schuman E (1999) Neurotrophin regulation of synaptic transmission. Curr Opin Neurobiol 9:105-109.

Segal MM, Furshpan EJ (1990) Epileptiform activity in microcultures containing small numbers of hippocampal neurons. J Neurophysiol 64:1390-1399.

Seil FJ (1999) BDNF and NT-4, but not NT-3, promote development of inhibitory synapses in the absence of neuronal activity. Brain Res 818:561-564.

Seil FJ, Drake-Baumann R (1994) Reduced cortical inhibitory synaptogenesis in organotypic cerebellar cultures developing in the absence of neuronal activity. J Comp Neurol 342:366-377.

Seil FJ, Drake-Baumann R, Leiman AL, Herndon RM, Tiekotter KL (1994) Morphological correlates of altered neuronal activity in organotypic cerebellar cultures chronically exposed to anti-GABA agents. Dev Brain Res 77:123-132.

Shieh PB, Hu S-C, Bobb K, Timmusk, Ghosh A (1998) Identification of a signaling pathway involved in calcium regulation of BDNF expression. Neuron 20:727-740.

Steward O, Falk PM (1991) Selective localization of polyribosomes beneath developing synapses: a quantitative analysis of the relationships between polyribosomes and developing synapses in the hippocampus and dentate gyrus. J Comp Neurol 314:545-557.

Stone DJ, Walsh J, Benes FM (1999) Localization of cells preferentially expressing GAD67 with negligible GAD65 transcripts in the rat hippocampus. A double in situ hybridization study. Mol Brain Res 71:201-209.

Stoppini L, Buchs P-A, Muller D (1991) A simple method for organotypic cultures of nervous tissue. J Neurosci Methods 37:173-182.

Tao X, Finkbeiner S, Arnold DB, Shaywitz AJ, Greenberg ME (1998) $\mathrm{Ca}^{2+}$ influx regulates BDNF transcription by a CREB family transcription factor-dependent mechanism. Neuron 20:709-726.

Thoenen H (1995) Neurotrophins and neuronal plasticity. Science 270:593-598.

Turrigiano GG, Leslie KR, Desai NS, Rutherford LC, Nelson SB (1998) Activity-dependent scaling of quantal amplitude in neocortical neurons. Nature 391:892-896.

Vicario-Abejón C, Collin C, McKay RDG, Segal M (1998) Neurotrophins induce formation of functional excitatory and inhibitory synapses between cultured hippocampal neurons. J Neurosci 18:7256-7271.

Zafra F, Castrén E, Thoenen H, Lindholm D (1991) Interplay between glutamate and gamma-aminobutyric acid transmitter systems in the physiological regulation of brain-derived neurotrophic factor and nerve growth factor synthesis in hippocampal neurons. Proc Natl Acad Sci USA 88:10037-10041.

Zafra F, Lindholm D, Castrén E, Hartikka J, Thoenen H (1992) Regulation of brain-derived neurotrophic factor and nerve growth factor mRNA in primary cultures of hippocampal neurons and astrocytes. J Neurosci 12:4793-4799. 